\title{
A Chinese version of the Psychotic Symptom Rating Scales: psychometric properties in recent-onset and chronic psychosis
}

This article was published in the following Dove Press journal:

Neuropsychiatric Disease and Treatment

8 March 2017

Number of times this article has been viewed

\author{
Wai-Tong Chien' \\ Isabella Yuet-Ming Lee ${ }^{2}$ \\ Li-Qun Wang ${ }^{3}$ \\ 'School of Nursing, Faculty of \\ Health and Social Sciences, The \\ Hong Kong Polytechnic University, \\ Hung Hom, Kowloon, Hong Kong, \\ People's Republic of China; ${ }^{2}$ Central \\ Nursing Division, Ruttonjee Tang \\ Shiu King Hospital, Wan Chai, Hong \\ Kong, People's Republic of China; \\ ${ }^{3}$ School of Nursing, Jilin Medical \\ College, Jilin City, Jilin Province, \\ People's Republic of China
}

\begin{abstract}
The purpose of this study was to test the reliability, validity, and factor structure of a Chinese version of the Psychotic Symptom Rating Scale (PSYRATS) in 198 and 202 adult patients with recent-onset and chronic psychosis, respectively. The PSYRATS has been translated into different language versions and has been validated for clinical and research use mainly in chronic psychotic patients but not in recent-onset psychosis patients or in Chinese populations. The psychometric analysis of the translated Chinese version included assessment of its content validity, semantic equivalence, interrater and test-retest reliability, reproducibility, sensitivity to changes in psychotic symptoms, internal consistency, concurrent validity (compared to a valid psychotic symptom scale), and factor structure. The Chinese version demonstrated very satisfactory content validity as rated by an expert panel, good semantic equivalence with the original version, and high interrater and test-retest (at 2-week interval) reliability. It also indicated very good reproducibility of and sensitivity to changes in psychotic symptoms in line with the symptom severity measured with the Positive and Negative Syndrome Scale (PANSS). The scale consisted of four factors for the hallucination subscale and two factors for the delusion subscale, explaining about $80 \%$ of the total variance of the construct, indicating satisfactory correlations between the hallucination and delusion factors themselves, between items, factors, subscales, and overall scale, and between factors and relevant item and subscale scores of the PANSS. The Chinese version of the PSYRATS is a reliable and valid instrument to measure symptom severity in Chinese psychotic patients complementary to other existing measures mainly in English language.
\end{abstract}

Keywords: delusion, hallucination, psychosis, PSYRATS, reliability, validity

\section{Introduction}

Schizophrenia and other psychotic disorders are chronic remitting and disruptive mental illnesses. In developed countries, psychotic patients constitute $40 \%-70 \%$ of the total patient population served by community mental health services. ${ }^{1}$ Psychotic symptoms, particularly auditory hallucination and delusions, are often disturbing, undesirable, and persistent; however, they are the key features or characteristics of psychoses. Difficulties in recovery from psychotic disorders have been closely associated with poor control and instability during these psychotic symptoms as well as nonadherence to medication and/or treatments. ${ }^{2,3}$ Very few structured assessment tools measure single or multiple domains/dimensions of psychotic symptoms, for example, Oulis Auditory Hallucinations Rating Scale ${ }^{4}$ and Delusions Inventory ${ }^{5}$ for single symptoms, or Positive and Negative Syndrome Scale (PANSS) ${ }^{6}$ and Revised Behavior and Symptom Identification Scale ${ }^{7}$ for overall symptoms. Among those
Correspondence: Wai-Tong Chien School of Nursing, Faculty of Health and Social Sciences, PQ 402, The Hong Kong Polytechnic University, Hung Hom, Kowloon, Hong Kong, People's Republic of China

Tel +85227665648

Fax +852 23649663

Email wai.tong.chien@polyu.edu.hk 
measures of psychotic symptoms, the Psychotic Symptom Rating Scales (PSYRATS) ${ }^{8}$ (Figure S1) consists of the multidimensional features of two main psychotic symptoms (ie, auditory hallucinations and delusions) in one measurement tool, and the PSYRATS has been translated into different languages, validated, and increasingly used for outcome evaluation of interventions for patients with acute and chronic psychotic disorders. ${ }^{9,10}$

The PSYRATS is an instrument conducted with semistructured interviews to measure the subjective characteristics of auditory hallucinations and delusions in multiple dimensions, including their nature, persistence, amount, severity, and beliefs toward the symptoms. ${ }^{8}$ It has often been used in mental health research and practice settings focusing on the two cardinal symptoms of schizophrenia and psychotic disorders on top of the overall assessment of psychotic symptoms (such as using the PANSyndrome Scale). This scale can assess more comprehensively the nature and severity of auditory hallucinations and delusions and thus provide more precise and accurate measurements on these two psychotic symptoms than other instruments for assessing specific aspects of voices (eg, the Revised Beliefs About Voices Questionnaire ${ }^{11}$ and the Voices Acceptance and Action Scale ${ }^{12}$ ) and specific types of delusions (eg, Paranoid Thought Scales ${ }^{13}$ and Paranoia Scale ${ }^{14}$ ).

As recommended by the original authors, ${ }^{8}$ the PSYRATS consists of 17 items assessing two subscales (auditory hallucinations and delusions), with each item being rated on a 5 -point scale $(0=$ Absent to $4=$ Severe/Often/Extreme). The scale has demonstrated satisfactory internal consistency (Cronbach's $\alpha=0.70-0.75),{ }^{15}$ interrater reliabilities (interclass correlation coefficient $($ ICC $)=0.79-0.90$ and $0.90-1.00$, respectively), ${ }^{8,16}$ test-retest reliabilities (ICC $=0.50-0.74$ at 7-day interval), ${ }^{16}$ and item-to-subscale and item-to-item correlations (Pearson's $r=0.37-0.77, P=0.01-0.001$ and $r=0.49-0.54, P=0.001$, respectively $)^{8,15,16}$ in people with psychotic disorders in a few English speaking countries. The auditory hallucinations subscale (AHS) contains 11 items: frequency, duration, loudness, origin, amount and intensity of distress and negative contents, beliefs about the origin of voices, disruption to life, and controllability. The delusions subscale (DS) consists of six items: amount and duration of preoccupation; conviction, amount, and intensity of distress; and disruption to life. Several studies on its construct validity using exploratory factor analysis identified 3-4-factor solutions within the two subscales. ${ }^{15-17}$ The original authors ${ }^{8}$ and Drake et al ${ }^{16}$ suggested the PSYRATS contained five dimensions using the multidimensional scaling method (Euclidean matrices, alternating least-squares algorithm [ALSCAL] subroutine), in which the AHS distress and negative content items loaded onto one factor; frequency and duration loaded onto another one; and belief about voices, location, control, and disruption loaded onto the third dimension. However, the DS frequency, duration, conviction, and disruption items loaded onto one factor, and the amount and intensity of distress loaded onto another factor. But, Kronmüller et $\mathrm{al}^{15}$ and Steel et $a{ }^{18}$ reported one additional dimension within the AHS items (controllability), thus including the four AHS dimensions (ie, emotional characteristics, physical characteristics, controllability, and cognitive interpretation). Validation studies on other language versions of the PSYRATS (eg, French, Indonesian, and German) supported the similar factor structure of the DS to the original study but could not find a clear-cut fourth factor structure (ie, controllability) for the AHS. ${ }^{15,19,20}$ With these differences on factor structure across countries, further construct validity testing on the PSYRATS is suggested.

There is a paucity of validation of the PSYRATS in recent-onset psychosis, except one conducted by Drake et al, ${ }^{16}$ and there is also a paucity in studies in Chinese patient populations. Variability in the course of schizophrenia among patients from different cultures has been observed. ${ }^{21-23}$ With inconsistencies on factor structure between countries and limited symptom measures in Chinese populations, a reliability and validity testing of a Chinese version of the PSYRATS is recommended to have better understanding of Chinese patients' needs for treatments or interventions. Therefore, this study was done to examine the psychometric properties of a Chinese version of the PSYRATS (C-PSYRATS) in recent-onset and chronic psychosis.

\section{Methods}

\section{Sample and study settings}

Patients with recent-onset ( $\leq 3$ year of the illness) and chronic ( $\geq 5$ years) psychosis were recruited from the outpatient and day-patient facilities in two regional mental hospitals (Kwai Chung and Kowloon hospitals) of the New Territories and Kowloon, which serve 1.5 million people (20\%) and take care of $40 \%$ of the psychotic patients in Hong Kong. These patients were recruited as part of three controlled trials to assess the effectiveness of mindfulness-based intervention, family self-help program, and adherence therapy in recent-onset psychosis and schizophrenia spectrum disorders. ${ }^{1,24,25}$ Ethical approvals to conduct the study were obtained from the Human Subjects Research Ethics Committee of The Hong Kong Polytechnic University (HSEARS2014121103) and Clinical Research Ethics Committee of the two hospital 
clusters of Hospital Authority Hong Kong (ie, the Kowloon Central and West clusters). Written informed consent was obtained from individual patients for participation after full explanation of the study purpose and procedure.

Inclusion criteria of the participants were: Hong Kong Chinese residents, aged 18-65 years, and diagnosed with schizophrenia and its subtypes and other psychotic disorders (onset $\leq 3$ years or $\geq 5$ years) according to the criteria of DSM-IV. ${ }^{26}$ Exclusion criteria were: comorbidities of organic brain disorder and learning disability, psychosis due to substance abuse, language and communication difficulties, or having been judged by psychiatrist as mentally unfit to participate. At least 170 patients with recent-onset and chronic psychosis were recruited in order to ensure adequate sample size for exploratory and confirmatory factor analysis ( $\geq 10$ subjects per item). ${ }^{27}$ Two subgroups of these participants (both $\mathrm{n}=50$ ) was selected; one subgroup was randomly selected (from the patient list in alphabetical order of their surnames using computer-generated random numbers) to assess test-retest reliability of the C-PSYRATS and another subgroup was purposively selected (ie, $>4$ scores in hallucination and delusion items of the PANSS) to assess its interrater reliability.

\section{Procedure}

\section{Translation and back-translation}

Translation of the English version of the PSYRATS into Chinese language followed the procedure of Brislin's mode ${ }^{28}$ in order to develop a culturally equivalent instrument. The original English version was translated into Chinese language by two bilingual mental health nurses independently and then back-translated into English by a second bilingual translator. The meaning of the original scale items was compared with the back-translated version among the translators and two researchers.

\section{Content validity and semantic equivalence}

Following the back-translation process, the bilingual version of the PSYRATS was administered to a panel of 10 persons who were experts in the study topic, including three psychiatrists, two advanced practice psychiatric nurses, two clinical psychologists, and three bilingual speaking ex-patients to determine the content validity (using Content Validity Index) and semantic equivalence (using intracorrelation test) of the Chinese version of the C-PSYRATS. The panel rated the semantic equivalence of the C-PSYRATS items to the meaning of the items in the original English version using a 4-point Likert scale, including 1 (not appropriate),
2 (somewhat appropriate), 3 (appropriate), and 4 (very appropriate). The panel was also invited to comment on the clarity and comprehensibility of the items and overall presentation and instructions of the instrument. The members of the panel were also asked to independently rate the relevance of the content of the C-PSYRATS items and the overall scale to local clinical context on a 4-point Likert scale: 1 (not relevant), 2 (somewhat relevant), 3 (relevant), and 4 (very relevant). As suggested by Polit and Beck, ${ }^{29}$ content validity index (CVI) of each item was determined by the proportion of experts who rated it as content valid (ie, rating of 3 or 4), and the CVI of the overall scale was determined by the proportion of all items $(\geq 80 \%)$ that were rated as content valid, for measuring the psychotic symptoms in local settings.

\section{Validity testing}

Patients were assessed within 5 days and reassessed 5 months later with the PANSS ${ }^{6}$ and C-PSYRATS by two psychiatrists (trained to administer the two scales) for concurrent validity, construct validity (exploratory and confirmatory factor analysis using the data from recent-onset and chronic psychosis, respectively), reproducibility, and sensitivity to changes in psychotic symptoms. The widely used PANSS had the most similar construct of psychotic symptoms to the PSYRATS, thus being most appropriate to make comparisons between their scores for validity testing.

Reproducibility of the C-PSYRATS was assessed with random-effects one-way ANOVA test on the data from those who reported no major changes in positive symptoms (PANSS scores) between the two measurements (over 5 months), with ICC of $\geq 0.7$ indicating satisfactory reproducibility. ${ }^{27}$ Responsiveness to change of the C-PSYRATS was assessed on the observed change in its two subscale scores over 5 months (ie, change divided by the baseline score minus the minimum possible score) to determine whether the mean change scores correlated with the PANSS hallucination/delusion item, positive symptom subscale, and/or total score.

\section{Test-retest and interrater reliability}

Sixty of the participants (30 from both recent-onset and chronic psychosis) were assessed with the C-PSYRATS at a 14-day interval to establish test-retest reliability, since these symptom scores were relatively stable over $1-2$ weeks. ${ }^{8}$ Another 60 participants who had scored 5 or more on the hallucination and delusion items of the PANSS were purposively selected to assess interrater reliability of the C-PSYRATS rated by three trained psychiatrists. 


\section{Internal consistency}

Internal consistency of the Chinese version and its subscales (refined after factor analysis) was tested by calculating the Cronbach's $\alpha$ coefficients, which indicated the homogeneity of construct of the C-PSTRATS.

\section{Results}

\section{Study participants}

One hundred ninety-eight patients with recent-onset psychosis and 202 chronic psychotic patients were recruited, and $120(61 \%)$ and 108 (54\%) were followed-up at 5 months, respectively (as part of the controlled trials by the research team). ${ }^{1,24,25}$ There were no significant differences on the demographic and clinical characteristics and symptom severity between the participants and nonparticipants at the two measurements (using $\chi^{2}$ or unpaired $t$-test; $P>0.15$ ). Overall, more than half of the 400 patients were male $(n=220,55 \%)$, and their mean age was 27.8 years (standard deviation $=10.7$ ). Majority of them were ethnic Chinese, either born in Hong Kong $(n=321,80 \%)$ or from mainland China ( $\mathrm{n}=58,15 \%)$, and were educated to a secondary school level or above $(n=322,81 \%)$. More than two-thirds $(n=258$, $65 \%$ ) were living with family members, and one-third had comorbidity with other mental illness such as mood disorders $(n=39,10 \%)$, anxiety disorders $(n=28,7 \%)$, and substance abuse $(n=31,8 \%)$.

\section{Face validity, semantic equivalence, and content validity}

The research team discussed about the discrepancies on the definitions of any items between the translated Chinese, back-translated English and original English versions of the PSYRATS and then clarified these with the translators. We had modified the wordings of only a few descriptions of the rating scales to ensure the congruence of meaning between the Chinese and English version, including "swear words" and "the milkman's ugly" in item 7, AHS (Degree of negative content), "supported accommodation" in item 10, AHS (Disruption to life by voices) and item 6, DS (Disruption to life by beliefs), and "dismiss or bring on them" in item 11, AHS (Controllability). Otherwise, there were no amendments to the items and headings. The translated Chinese version is presented in Figure S2.

The intraclass correlation coefficients between the Chinese and the original English version were $0.92(P=0.005)$ for the overall scale and from 0.88 to 0.98 for the two subscales. Very minor amendments to the wordings of a few items were made (eg, "majority" and "minority" of voices, "conviction" to voices and "preoccupation" with delusions).
The C-PSYRATS also showed very satisfactory content validity, with the item-level CVIs between 0.92 and 1.00 and scale-level CVI of 0.98 .

\section{Interrater reliability}

ICCs between three raters for subscale and total scores of the C-PSYRATS in recent-onset psychosis were very satisfactory, with a value of 0.92 for AHS, 0.95 for DS, and 0.94 for the overall scale. The ICCs between raters ranged 0.82-1.00 for AHS items and 0.80-1.00 for DS items, except 0.50 for "location of voices". The ICCs between raters in chronic psychosis were also very satisfactory, with values of 0.80-1.00 for AHS and overall scale and 0.78-0.96 for DS, except that it was 0.42 for "location of voices". There were small discrepancies $(2 \%-6 \%)$ between raters on the C-PSYRATS subscale/total scores in recent-onset and chronic psychosis.

\section{Test-retest reliability}

Data from the 60 participants after a 14-day interval were compared, and the ICCs were 0.81 and 0.82 for AHS and 0.85 and 0.81 for DS in recent-onset and chronic psychosis, respectively. The ICCs for individual AHS and DS items in recent-onset psychosis were $0.76-0.88$ and $0.80-0.90$, respectively, whereas the ICCs for AHS and DS items were $0.72-0.82$ and $0.76-0.85$, respectively, in chronic psychosis. Over the 14 days of measurement, there were very minimal changes detected in levels of positive symptoms (ie, 0.2-0.9 or $0.4 \%-2.5 \%$ of changes in positive symptom subscale of the PANSS) among both illness groups.

\section{Concurrent validity}

In recent-onset psychosis, both the AHS and DS significantly correlated with the PANSS hallucination/delusion item (Pearson's $r=0.56$ and 0.68 and $P=0.007$ and 0.002 , respectively), positive symptom subscale ( $r=0.32$ and 0.38 and $P=0.03$ and 0.02 , respectively), and total score ( $r=0.26$ and 0.29 and $P=0.05$ and 0.04 , respectively). Similarly, both the AHS and DS in chronic psychosis significantly correlated with the PANSS hallucination/delusion item $(r=0.54$ and 0.62 and $P=0.008$ and 0.005$)$, positive symptom subscale $(r=0.35$ and 0.38 and $P=0.02$ and 0.01 ), and total score $(r=0.29$ and 0.32 and $P=0.04$ and 0.03 ).

\section{Reproducibility and sensitivity to changes in symptom severity}

Reproducibility of the C-PSYRATS between the 5-month measurements was found to have ICCs of $0.86(F=7.25$, 
$P=0.003)$ in recent-onset psychosis and $0.78(F=5.60$, $P=0.01$ ) for chronic psychosis, indicating a very satisfactory consistency in testing the psychotic symptoms of those mentally stable patients in the two illness groups using the C-PSYRATS.

The observed changes in the AHS, DS, and total score of the C-PSYRATS were 1.1, 1.2, and 2.0 in recent-onset psychosis and 1.2, 1.3, and 2.2 in chronic psychosis, accordingly. Change in the AHS significantly correlated with the changes in the PANSS hallucination item (Spearman's $r=0.70$ and $0.63, P=0.005$ and 0.009 ), positive symptom subscale ( $r=0.60$ and $0.52, P=0.01$ and 0.03 ), and total score ( $r=0.66$ and $0.53, P=0.007$ and 0.02 ) in both illness groups. Similarly, change in the DS significantly correlated with that in the PANSS delusion item ( $r=0.81$ and $0.79, P=0.001$ and 0.002 ), positive symptom subscale $(r=0.64$ and $0.56, P=0.008$ and 0.02 ), and total score ( $r=0.68$ and $0.57, P=0.005$ and 0.02 ) in both illness groups.

\section{Construct validity}

Principal components analysis followed by oblique rotation was performed for the data from 198 patients with recent-onset psychosis, using SPSS for Windows version 20.0 (SPSS Inc., Chicago, IL, USA). The data indicated satisfactory item-total correlations $(>0.30)$, adequate sample size, and factorability (ie, Kaiser-Meyer-Oklin value of 0.84 and Bartlett test of Sphericity $\left.\chi^{2}=1,304.3, d f=194, P<0.001\right)$ for factor analysis. ${ }^{27}$ Considering the Catell's scree plot (Figure 1), six principal factors were initially extracted with $>1.0$ in the unrotated matrix. All 17 items met the criterion on factor loading of $>0.40$, and were thus retained for oblique rotation. ${ }^{30}$ The rotated solution revealed that each of the six factors contained of a few items, which were loaded

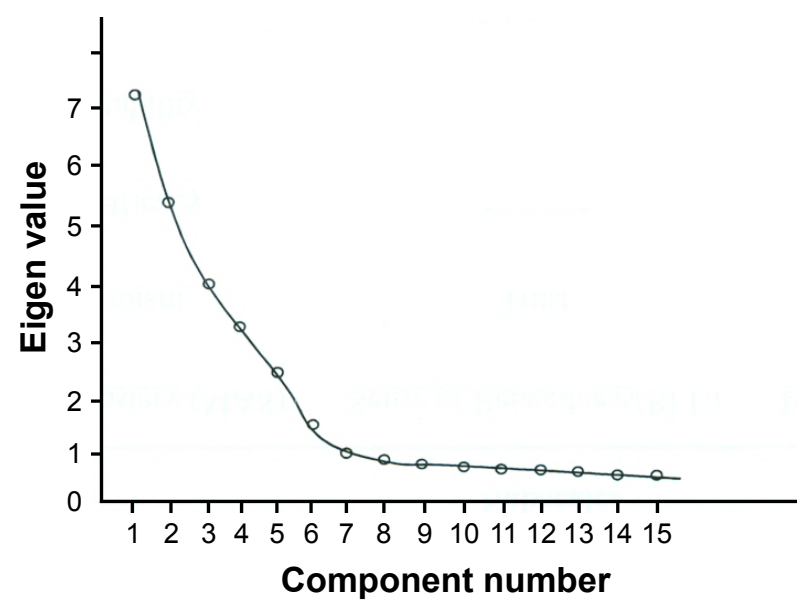

Figure I Catell's scree plot in exploratory factor analysis. substantially $(>0.65)$ on one factor only. The results of the oblique rotation are presented in Table 1. The six factor solutions included: four for the AHS, 1) amount and degree of negative content and distress, 2) frequency and duration, 3) loudness, disruption, and controllability, and 4) location and beliefs regarding origin; and two for the DS, 5) amount and duration of preoccupation and conviction, and 6) amount and intensity of distress and disruption to life (by beliefs). These six factors explained $80.1 \%$ of the total variance of the symptom construct in recent-onset psychosis. The six factors also moderately to highly correlated with the C-PSYRATS total score (Pearson's $r=0.48-0.80, P=0.01-0.001$ ) and were moderately intercorrelated $(r=0.33-0.76, P=0.05-0.005)$.

Confirmatory factor analysis (using the LISREL 9.1 software, Scientific Software International, Skokie, IL, USA) on the data collected from the 202 participants with chronic psychosis was then performed to compare between the six factors identified from the exploratory factor analysis and those (five-factor model) suggested by the original authors, ${ }^{8}$ in order to determine the maximum likelihood "goodness-offit" of these predetermined factor models. A summary of the Fit indices of the two models of the C-PSYRATS with both uncorrelated and correlated factors is shown in Table 2.

The first model (Table 2) identified from those with recent-onset psychosis appears to fit the data very well. The six-factor model with paths between all factors showed very good fit according to all fit indices $\left(\chi^{2} / d f=2.14, P=0.54\right.$; adjusted goodness of fit $[\mathrm{AGFI}]=0.97$; Tucker-Lewis index $[\mathrm{TLI}]=1.04$; root mean square error of approximation $[$ RMSEA] $=0.034$; weighted root mean residual [WRMR] $=0.79$ ), indicating that it was better than the original five-factor model. Critical ratios for the regression weights were all $>2.5$ (ie, each item had a significant contribution to its correlated factor). Indeed, the original fivefactor model indicated an acceptable fit $\left(\chi^{2} / d f=1.96, P=0.32\right.$; AGFI $=0.90 ;$ TLI $=0.96$; RMSEA $=0.049$; WRMR $=0.88$ ). In addition, model modification indices for the six-factor model indicated good fits, particularly when additional paths were drawn between factors. The path diagram showed that there were moderate to moderate-high correlations between factors and corresponding items (Pearson's $r=0.48-0.75, P=0.02-0.003)$ and between the factors themselves $(r=0.38-0.82, P=0.01-0.001)$.

\section{Internal consistency}

Cronbach's $\alpha$ coefficients of the Chinese version (after factor analysis) were 0.89 for the overall scale and 0.86 and 0.90 for the AHS and DS, respectively, indicating very good 
Table I Results of oblique rotation of four factor solutions for C-PSYRATS in recent psychosis (N=198)

\begin{tabular}{|c|c|c|c|c|c|c|}
\hline \multirow[t]{2}{*}{ Items } & \multicolumn{6}{|c|}{ Factor loading } \\
\hline & Factor I & Factor 2 & Factor 3 & Factor 4 & Factor 5 & Factor 6 \\
\hline I. $\mathrm{AH}$ - Frequency & & 0.88 & & & & \\
\hline 2. $\mathrm{AH}$ - Duration & & 0.87 & & & & \\
\hline 3. $\mathrm{AH}$ - Location & & & & 0.73 & & \\
\hline 4. $\mathrm{AH}$ - Loudness & & & 0.70 & & & \\
\hline 5. $\mathrm{AH}-$ Beliefs regarding origin of voices & & & & 0.80 & & \\
\hline 6. $\mathrm{AH}-$ Amount of negative content & 0.68 & & & & & \\
\hline 7. $\mathrm{AH}$ - Degree of negative content & 0.72 & & & & & \\
\hline 8. $\mathrm{AH}-$ Amount of distress & 0.80 & & & & & \\
\hline 9. $\mathrm{AH}-$ Intensity of distress & 0.82 & & & & & \\
\hline 10. AH - Disruption to life caused by voices & & & 0.80 & & & \\
\hline II. AH - Controllability & & & 0.88 & & & \\
\hline 12. D - Amount of preoccupation & & & & & 0.88 & \\
\hline 13. D - Duration of preoccupation & & & & & 0.89 & \\
\hline I4. D - Conviction & & & & & 0.84 & \\
\hline 15. D - Amount of distress & & & & & & 0.80 \\
\hline 16. D - Intensity of distress & & & & & & 0.90 \\
\hline 17. D - Disruption to life & & & & & & 0.89 \\
\hline Eigen value & 7.75 & 2.31 & 3.59 & 1.48 & 4.90 & 5.21 \\
\hline Percentage of variance explained & 18.02 & 9.40 & 13.76 & 9.51 & 14.55 & 14.82 \\
\hline
\end{tabular}

Notes: Factor loadings $\geq 0.40$ are reported. For AH, Factor I= Emotional; Factor $2=$ Physical; Factor $3=$ Control; Factor $4=$ Cognitive. For $\mathrm{D}$, Factor $5=$ Cognitive; Factor 6= Emotional.

Abbreviations: C-PSYRATS, Chinese version of Psychotic Symptom Rating Scales; AH, auditory hallucination subscale; D, delusion subscale.

homogeneity of the construct(s) of the C-PSYRATS in recent-onset psychosis. The $\alpha$ coefficients of the C-PSYRATS in chronic psychosis were $0.87,0.84$, and 0.89 for the overall scale, AHS, and DS, accordingly.

\section{Discussion}

This study examined the psychometric properties of a Chinese version of the PSYRATS (C-PSYRATS) in both recent-onset and chronic psychosis. The time for completion of the scale was acceptable, lasting about 10 minutes. The C-PSYRATS demonstrated very similar conceptual meanings of the items to the original version and was relevant and appropriate to assess the psychotic symptoms as indicated by very good results of content validity and semantic equivalence.
The overall scale and its two subscales (AHS and DS) showed high Cronbach's $\alpha$ values, indicating very satisfactory internal consistency or homogeneity of the construct of the Chinese version. The high intraclass correlations of the repeated and between-assessor ratings of the 60 psychotic patients indicated a high stability of the C-PSYRATS over a reasonable 2-week interval and between trained raters. The ratings on the item for the location of voices much varied between the assessors and this might be due to the differences on the patients' personal attributions of meaning to their experiences of the voices; and thus they hesitated to inform whether the voices generated from inside or outside the head or self, as suggested by the Hearing Voices Movement. ${ }^{31}$ Such patients' challenge and difficulty in appraising their relationship with

Table 2 Summary of fit indices of two hypothesized models of C-PSYRATS ( $N=202)$

\begin{tabular}{|c|c|c|c|c|c|c|c|c|c|c|}
\hline Model & $\chi^{2}$ & $d f$ & $\chi^{2} / d f$ & $P$-value & GFI & AGFI & TLI & RMSEA $(90 \% \mathrm{Cl})$ & SRMR & WRMR \\
\hline \multicolumn{11}{|l|}{ Six-factor model } \\
\hline Uncorrelated factors & 68.98 & 30 & 2.30 & 0.56 & 1.00 & 0.95 & 1.00 & $0.036(0.028-0.042)$ & 0.022 & 0.82 \\
\hline Correlated factors $\mathrm{s}^{\mathrm{a}}$ & 62.10 & 29 & 2.14 & 0.54 & 0.99 & 0.97 & 1.04 & $0.034(0.027-0.040)$ & 0.019 & 0.79 \\
\hline \multicolumn{11}{|l|}{ Five-factor model } \\
\hline Uncorrelated factors & 70.65 & 37 & 1.91 & 0.30 & 0.90 & 0.89 & 0.91 & $0.047(0.040-0.054)$ & 0.046 & 0.89 \\
\hline Correlated factors & 68.63 & 35 & 1.96 & 0.32 & 0.91 & 0.90 & 0.96 & $0.049(0.038-0.058)$ & 0.048 & 0.88 \\
\hline
\end{tabular}

Notes: Six-factor model identified in this research in recent-onset psychosis; Five-factor model suggested by the original authors $\left(\mathrm{Haddock}\right.$ et al $\left.{ }^{8}\right)$. ${ }^{\mathrm{P}} \mathrm{Codel}$ fit indices tested with paths (correlations) set up between the hypothesized factors in each model. $\chi^{2}=$ Chi-squared goodness-of-fit; $d f=d e g r e e$ of freedom; $P$-value (a good fit if $P \geq 0$. I). Abbreviations: GFI, goodness-of-fit index (range 0-I, a good-fit if GFI $\geq 0.9$ ); AGFI, adjusted good-of-fit index (similar to GFI, a good-fit if AGFI $\geq 0.9$ ); TLI, Tucker-Lewis index (0.90-0.95 acceptable, a good-fit if TLI >0.95); RMSEA, root mean square error of approximation (a good-fit if RMSEA $\leq 0.05$ ); SRMR, standardized root mean square residual (a good-fit if SRMR <0.05); WRMR, weighted root mean residual (a good-fit if WRMR $<0.90$ ). 
the voices should be further explored in order to better understand patients' personal meaning assigned to the voices, thus leading to better coping with this symptom.

Moreover, the C-PSYRATS demonstrated significant association (high concurrent validity) with the widely used psychotic symptom scale (PANSS) regarding hallucinations and delusions. The overall and subscale scores of the C-PSYRATS indicated very satisfactory reproducibility and sensitivity to change in psychotic symptoms (PANSS total and subscale scores) in both recent-onset and chronic psychosis over 5 months. These results support the C-PSYRATS to be a valid instrument or outcome measure to measure the symptom severity among these psychotic patients across time, as well as in terms of various dimensions (eg, amount, duration and level of disruption) of hallucinations and delusions.

The exploratory factor analysis demonstrated a six-factor structure of the C-PSYRATS, which is similar to the ones suggested by Kronmüller et a ${ }^{15}$ and Steel et al, ${ }^{18}$ and more importantly, these factors explained very high percentage $(>80 \%)$ of the total variance of the symptom construct in recent-onset psychosis. For auditory hallucination subscale (AHS), two items (disruption to life and control) from cognitive interpretation and one item (loudness) from physical characteristics suggested by the original authors were loaded onto one additional factor termed "controllability". Such inconsistencies in factor structure of the AHS reveal the lack of clear understanding about the complex nature and heterogeneity of hallucinatory experiences or dimensions. ${ }^{20}$ As suggested by Steel et al, ${ }^{18}$ the negative impacts (disruptions) of voices on patients' life are strongly connected with the loudness of and control over their voices experienced, which sometimes improves earlier than other dimensions of the hallucinations. ${ }^{32}$ With respect to the delusion subscale (DS), the two dimensions identified are consistent with the findings of most previous studies, ${ }^{18}$ even though disruption to life was loaded onto amount, duration, and conviction of delusions in two previous studies. ${ }^{8,15,16}$

The six-factor model of the C-PSYRATS identified from recent-onset psychosis was found the best fit in the confirmatory factor analysis for chronic psychosis. Therefore, the six dimensions of psychotic symptoms (AHS and DS) can better explain the symptom construct in two different psychotic patient populations than the original five-factor model proposed by Haddock et al. ${ }^{8}$ It is important and useful to confirm these dimensions of AHS and DS in the C-PSYRATS because these item clusters may reflect the underlying etiological process of psychosis. ${ }^{17}$ For instance, the amount and duration of preoccupation and conviction items (plus the disruption item) have clustered into one dimension (factor) of the DS. This may reflect that delusion might be affected by cognitive bias in which the false beliefs persist against evidence of disconfirmation or easily tend to jump into a subjective biased conclusion..$^{33}$ The amount and degree of negative content and distress could co-occur and be interconnected and thus represent the emotional reactions toward the voices. This can help distinguish clinically diagnosed psychotic patients from nonclinically diagnosed voice-hearing persons. ${ }^{34}$

There were a few main limitations of this study. The study sample were those participated in one of the three controlled trials conducted by the first author. These samples, recruited from two of 14 psychiatric outpatient and day care facilities, might not be representative to the psychotic patient population in Hong Kong. Those with acute psychotic symptoms and/or lack of insight into the illness could not have been included into this study. In addition, the sample was selective because most of the participants were well educated and relatively mentally stable Hong Kong-born Chinese people. Therefore, these results are in need of replication in psychotic patients at psychiatric hospital and rehabilitation settings and in those in different stages of psychosis. It is also noteworthy that the PSYRATS was developed to mainly measure auditory hallucinations and all delusions in general, which cannot fully represent the variety of psychotic symptoms or symptom severity. As suggested by a critical review on treatment targets in schizophrenia, ${ }^{35}$ it is of high relevance to capture other important domains than these two cardinal symptoms, such as negative and cognitive symptoms and even subjective experiences of the illness, as what constitutes an acceptable clinical status is hard to define and reasonably target to evaluate for an effective treatment regimen in clinical settings. It is also unknown whether the identified dimensions of the C-PSYRATS can represent exhaustively all important dimensions of hallucinations and delusions, particularly due to the fact that development of the scale was based on interviews with patients with persistent auditory hallucination. ${ }^{8}$ A future study can investigate how the overall scale, two subscales, and identified dimensions of the C-PSYRATS are associated with demographic and illness-related correlates and specific responses to psychotic symptoms such as mindfulness, acceptance, or omnipotence of beliefs about hallucinations.

\section{Conclusion}

The C-PSYRATS has demonstrated very good reliability and validity in both recent-onset and chronic psychosis. It also indicated a high concurrent validity with the widely 
accepted psychotic symptom measure (PANSS). This scale has the advantage of easy administration with minimal training and provides detailed assessment of different dimensions of auditory hallucinations and delusions. The six dimensions of the scale identified can lead to better understanding of the underlying etiological processes of these psychotic symptoms and allow precise measurement of the changes in the corresponding symptom dimension(s). Further research is recommended to test the psychometric properties of the C-PSYRATS, particularly its predictive, discriminatory, and construct validity, in larger samples of Chinese patients with diverse sociodemographic, illness, and other clinical characteristics.

\section{Acknowledgments}

The authors thank the General Research Fund (PolyU 156038/15H), University Grant Council, The Hong Kong SAR's Government, for supporting this research project. We would also like to express our sincere thanks to the staff of the two outpatient and day-care facilities who kindly assisted and supported the subject recruitment and data collection.

\section{Disclosure}

The authors report no conflicts of interest in this work.

\section{References}

1. Chien WT, Liu JYW, Yip ALK, McMaster TW. The effectiveness of manual-guided, problem-solving-based self-learning programme for family caregivers of people with recent-onset psychosis: a randomised controlled trial with 6-month follow-up. Int J Nurs Stud. 2016;59: $141-155$.

2. Kern RS, Glynn SM, Horan WP, Marder SR. Psychosocial treatments to promote functional recovery in schizophrenia. Schizophr Bull. 2009;35:347-361.

3. Van Os J, Linscott RJ, Myin-Germeys I, Delespaul P, Krabbendam L. A systematic review and meta-analysis of the psychosis continuum: evidence for a psychosis proneness-persistence-impairment model of psychotic disorder. Psychol Med. 2009;39:179-195.

4. Oulis PG, Mavreas VG, Mamounas JM, Stefanis CN. The clinical characteristics of auditory hallucinations. Acta Psychiatr Scand. 1995; 92:97-102.

5. Peters ER, Joseph SA, Garety PA. The measurement of delusional ideation in the normal population: introducing the PDI (Peters et al Delusions Inventory). Schizophr Bull. 1999;25:553-576.

6. Kay SR, Opler LA, Lindenmayer JP. The positive and negative syndrome scale (PANSS): rationale and standardisation. Br J Psychiatry. 1989; 155(Suppl):59-67.

7. Eisen SV, Normand S-L, Belanger AJ, Spiro A III, Esch D. The revised behavior and symptom identification scale (BAIS-R): reliability and validity. Med Care. 2004;42(12):1230-1241.

8. Haddock G, McCarron J, Tarrier N, Faragher EB. Scales to measure dimensions of hallucinations and delusions: the psychotic symptom rating scales (PSYRATS). Psychol Med. 1999;29:879-889.

9. Kråkvik B, Gråwe RW, Hagen R, Stiles TC. Cognitive behaviour therapy for psychotic symptoms: a randomized controlled effectiveness trial. Behav Cogn Psychother. 2013;41:511-524.
10. Sommer IE, Daalman K, Rietkerk T, et al. Healthy individuals with auditory verbal hallucinations; who are they? Psychiatric assessments of a selected sample of 103 subjects. Schizophr Bull. 2010;36: 633-641.

11. Chadwick $P$, Lees $S$, Birchwood M. The revised beliefs about voices questionnaire (BAVQ-R). Br J Psychiatry. 2000;177:229-232.

12. Shawyer F, Ratcliff K, Mackinnon A, Farhall J, Hayes SC, Copolov D. The voice acceptance and action scale (VAAS): pilot data. $J$ Clin Psychol. 2007;63:593-606.

13. Green CE, Freeman D, Kuipers E, et al. Measuring ideas of persecution and social reference: the Green et al. Paranoid Thought Scales (GPTS). Psychol Med. 2008;38:101-111.

14. Fenigstein A, Vanable PA. Paranoia and self-consciousness. J Pers Soc Psychol. 1992;62:129-138.

15. Kronmüller KT, von Bock A, Grupe S, et al. Psychometric evaluation of the psychotic symptom rating scales. Compr Psychiatry. 2011;52: 102-108.

16. Drake R, Haddock G, Tarrier N, Bentall R, Lewis S. The Psychotic Symptom Rating Scales (PSYRATS): Their usefulness and properties in first episode psychosis. Schizophr Res. 2007;89:119-122.

17. Woodward TS, Jung K, Hwang H, et al. Symptom dimensions of the psychotic symptom rating scales in psychosis: a multisite study. Schizophr Bull. 2014;40(Suppl 4):265-274

18. Steel C, Garety D, Freeman E, et al. The multidimensional measurement of the positive symptoms of psychosis. Int J Methods Psychiatr Res. 2007; 16:88-96.

19. Erawati E, Keliat BA, Daulima NHC. The validation of the Indonesian version of the psychotic symptom rating scales (PSYRATS), the Indonesian version of bias questionnaire for psychosis (CBOP) and metacognitive ability questionnaire (MAQ). Int $J$ Adv Nurs Stud. 2014;3: $97-100$.

20. Favrod J, Rexhaj S, Ferrari P, et al. French version validation of the psychotic symptom rating scales (PSYRATS) for outpatients with persistent psychotic symptoms. BMC Psychiatry. 2012;2:161.

21. Bentsen H, Notland TH, Munkvold O, et al. Guilt proneness and expressed emotion in relatives of patients with schizophrenia or related psychoses. Br J Med Psychol. 1998;71(Pt 2):125-138.

22. Phillips MR, Pearson V, Li F, Xu M, Yang L. Stigma and expressed emotion: a study of people with schizophrenia and their family members in China. Br J Psychiatry. 2002;181:488-493.

23. Weisman A, Rosales G, Kymalainen J, Armesto J. Ethnicity, family cohesion, religiosity and general emotional distress in patients with schizophrenia and their relatives. J Nerv Mental Dis. 2005; 193(6):359-368.

24. Chien WT, Mui JH, Cheung EF, Gray R. Effects of motivational interviewing-based adherence therapy for schizophrenia spectrum disorders: a randomized controlled trial. Trials. 2015;16:270.

25. Chien WT, Thompson DR. Effects of a mindfulness-based psychoeducation programme for Chinese patients with schizophrenia: two-year follow-up. Br J Psychiatry. 2014;205:52-59.

26. American Psychiatric Association. Diagnostic and Statistical Manual of Mental Disorders. 4th ed. Washington, DC: American Psychiatric Association Press; 1994.

27. Stevens J. Applied Multivariate Statistics for the Social Sciences. 4th ed. Mahwah, NJ: Lawrence Erlbaum; 2002.

28. Brislin RW. The wording and translation of research instruments. In: Lonner WJ, Berry JW, editors. Field Methods in Cross-cultural Research. Thousand Oaks, CA: Sage Publications, Inc.; 1986:137-164.

29. Polit DF, Beck CT. The content validity index: are you sure you know what's being reported? Critique and recommendations. Res Nurs Health. 2006;29:489-497.

30. Nunnally JC, Bernstein IH. Psychometric Theory. 3rd ed. New York, NY: McGraw-Hill; 1994

31. Corstens D, McCarthy-Jones S, Waddingham R, Thomas N. Emerging perspectives from the Hearing Voices Movement: implications for research and practice. Schizophr Bull. 2014;40(Suppl 4):285-294. 
32. Schneider SD, Jelinek L, Lincoln TM, Moritz S. What happened to the voices? A fine-grained analysis of how hallucinations and delusions change under psychiatric treatment. Psychiatry Res. 2011; 188(1):13-17.

33. Speechley WJ, Whitman JC, Woodward TS. The contribution of hypersalience to the "jumping to conclusions" bias associated with delusions in schizophrenia. J Psychiatry Neurosci. 2010;35:7-17.
34. Johns LC, Kompus K, Connell M, et al. Auditory verbal hallucinations in persons with and without a need for care. Schizophr Bull. 2014; 40(Suppl 4):255-264.

35. Suzuki T, Uchida H, Watanable K, Kashima H. Treatment target in schizophrenia: a critical review and a clinical suggestion. Psychopharmacol Bull. 2008;41:80-102.

\section{Publish your work in this journal}

Neuropsychiatric Disease and Treatment is an international, peerreviewed journal of clinical therapeutics and pharmacology focusing on concise rapid reporting of clinical or pre-clinical studies on a range of neuropsychiatric and neurological disorders. This journal is indexed on PubMed Central, the 'PsycINFO' database and CAS, and is the official journal of The International Neuropsychiatric Association (INA). The manuscript management system is completely online and includes a very quick and fair peer-review system, which is all easy to use. Visit http://www.dovepress.com/testimonials.php to read real quotes from published authors.

Submit your manuscript here: http://www.dovepress.com/neuropsychiatric-disease-and-treatment-journal 